\title{
Neue Aspekte in der Behandlung von Siedlungsabflüssen
}

\author{
Stephan Fuchs · Benedikt Lambert • Dieter Grotehusmann
}

Eingegangen: 19. Juli 2010/Akzeptiert: 10. September 2010/Online veröffentlicht: 7. Oktober 2010

(C) Springer-Verlag 2010

Zusammenfassung Die Siedlungsentwässerung ist mit hohen Frachtanteilen an der Schadstoffbelastung von Gewässersystemen beteiligt. Ausschlaggebend hierfür sind die über Mischwasserentlastungen und Regenwassereinleitungen eingetragenen Partikel, die als Transportmatrix für anthropogene Schadstoffe (z. B. Schwermetalle, MKW, PAK u. a.) im Siedlungsabfluss fungieren.

Zur Umsetzung effizienter Maßnahmen der Regenwasserbehandlung ist es erforderlich, die Feststoffe in eine grobe (Sand und Kies) und eine feine (Ton und Schluff) Fraktion zu unterteilen. Beide Fraktionen zeigen ein grundsätzlich unterschiedliches Systemverhalten in Bezug auf ihre Schadstoffbeladung sowie Transport- und Retentionsprozesse im Einzugsgebiet und Entwässerungssystem.

Eine primäre Anforderung an die Regenwasserbehandlung ist der wirksame Rückhalt der stark mit Schadstoffen beladenen Feinpartikel. Treten in nennenswertem Umfang Grobpartikel organischen Ursprungs auf, so sind auch sie eine Zielgröße. Das mineralische Grobkorn ist dagegen schadstoffarm und nur dann in die Maßnahmenplanung einzubeziehen, wenn die u. U. hohen Feststoffmassen Probleme

Verantwortliche Herausgeber: Organisationskomitee der Tagung

S. Fuchs $(\bowtie)$

Karlsruher Institut für Technologie (KIT),

Institut für Wasser und Gewässerentwicklung,

Bereich Siedlungswasserwirtschaft und Wassergütewirtschaft,

Adenauerring 20 b, 76128 Karlsruhe, Deutschland

E-Mail: stephan.fuchs@kit.edu

B. Lambert

Bioplan Landeskulturgesellschaft,

Pfohlhofstr. 20, 74889 Sinsheim, Deutschland

D. Grotehusmann

Ingenieurgesellschaft für Stadthydrologie,

Stiftsstraße 12, 30159 Hannover, Deutschland bereiten. In größeren Entwässerungsgebieten findet eine ausgeprägte Abreicherung der groben, mineralischen Fraktion statt. Die an der Einleitungsstelle bzw. am Behandlungsort ankommenden Feststoffe bestehen dann zu 70 bis $90 \%$ aus Feinpartikeln. Konventionelle Absetzanlagen mit Oberflächenbeschickungen zwischen 10 bis $2 \mathrm{~m} / \mathrm{h}$ sind bezüglich des Feinpartikelrückhalts weitgehend wirkungslos. Nur durch eine deutliche Reduzierung der Oberflächenbeschickung, z. B. durch den Einbau von Schrägklärelementen oder die Nutzung vorhandener Rückhalteräume, kann ein sicherer Feinpartikelrückhalt erreicht werden. Filtrationsanlagen sind per se hochwirksam. Ihr Einsatz ist im urbanen Raum aufgrund des hohen Flächenbedarfes allerdings limitiert.

Schlüsselwörter Behandlungsanlagen · Feinpartikel · Feststoffe $\cdot$ Schwermetalle

\section{Surface runoff from urban areas - new aspects}

Abstract The surface runoff from urban areas is one of the most important sources of pollutants emitted into surface waters. Suspended solids which act as a transport vehicle for many anthropogenic pollutants (e.g. heavy metals, PAH) are a key factor in this regard. The development of efficient measures of storm water runoff treatment thus requires a further differentiation of suspended solids in a fine (clay and silt) and coarse (sand and gravel) fraction. Both fractions show distinctly different characteristics in pollutant loading, transport and retention on urban surfaces and sewer systems. The primary aim of storm water runoff treatment is the reduction of the fine particles which are always highly loaded with anthropogenic pollutants. In contrast the coarse particles are almost unpolluted especially if they have a low organic share. The widespread sedimentation tanks with 
surface loadings between 10 and $2 \mathrm{~m} / \mathrm{h}$ are very inefficient. A significant, save and lasting reduction of the emitted load of fine particles requires a considerable reduction of the surface loads. That can be achieved with the installation of lamellar settler or the utilization of the very large volumes of flood management tanks frequently present in urban areas. Filtration plants are highly efficient but there application in urban areas is limited due to their high space demands.

Keywords Fine particles · Heavy metals $\cdot$ Storm water treatment $\cdot$ Total suspended solids

\section{Einleitung}

Die übergeordnete Zielsetzung der Regenwasserbehandlung oder -bewirtschaftung ist es, die Eingriffe in den natürlichen Wasserhaushalt von Einzugsgebieten in Bezug auf Menge und stoffliche Belastung so gering wie möglich zu halten. Aus dieser übergeordneten Zielsetzung leiten sich Handlungsbereiche mit unterschiedlicher zeitlicher und räumlicher Bedeutung ab:

- Reduzierung der hydraulischen Stoßbelastung von Gewässern,

- Reduzierung der Stoffeinträge mit dem Ziel der Einhaltung von gewässerverträglichen Grenzkonzentrationen und

- Reduzierung der Stoffeinträge mit dem Ziel einer Frachtminderung.

Während sich die erstgenannten Zielgrößen in der Regel aus der lokalen Gewässersituation ableiten, ist das letztgenannte Ziel im Zusammenhang mit Gewässersystemen resp. übergeordneten Schutzzielen (Meeresschutz) zu sehen.

Ein genereller Handlungsbedarf im Bereich der Regenwasserbehandlung leitet sich insbesondere für die Schwermetalle aus aktuellen Flussgebietsbilanzierungen ab. Mischwasserentlastungen und Regenwassereinleitungen sind mit hohen Massenanteilen an der gesamten in die Gewässer emittierten Schwermetallfracht beteiligt. Die aus diesen Eintragspfaden resultierenden Frachtanteile verursachen in einzelnen Flussgebieten mehr als $50 \%$ der Gesamteinträge (Fuchs et al. 2009a). Insbesondere in den Gebieten, die überwiegend im Trennsystem entwässert werden, wurden die höchsten Frachtanteile berechnet. Ursächlich hierfür sind die über die Stadtentwässerung eingetragenen, hohen Feststofffrachten.

\section{Feststoffe in Entwässerungssystemen}

Die Feststoffe, in der Regel als abfiltrierbare Feststoffe (AFS) gemessen, haben eine Schlüsselrolle in der Stadtentwässerung. Dies leitet sich zum einen daraus ab, dass sie mit

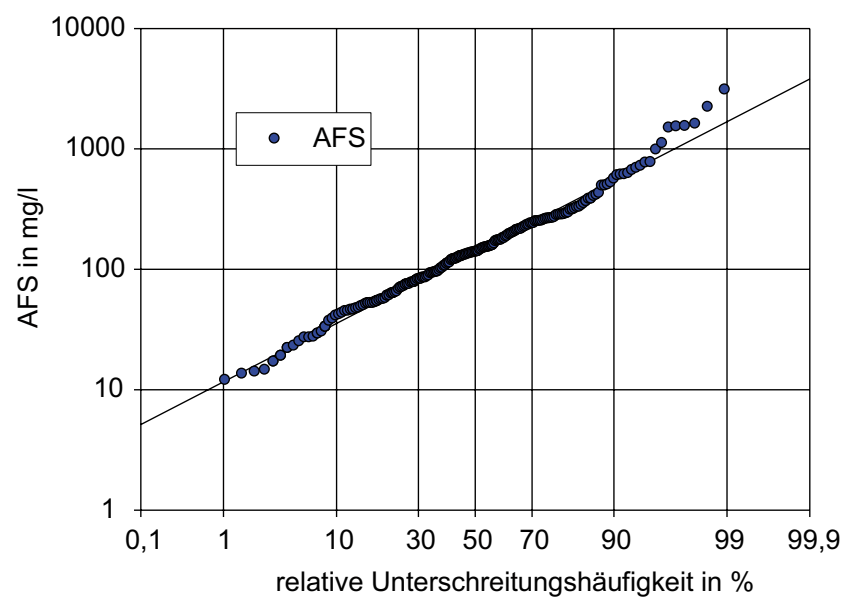

Abb. 1 Messdaten zu AFS-Konzentrationen im Regenabfluss (nach Brombach und Fuchs 2002, Fortschreibung)

den heute verfügbaren Instrumenten die primäre oder häufig auch einzige unmittelbar beeinflussbare Größe darstellen. Zum anderen agieren die Feststoffe im Entwässerungssystem als Transportmatrix für nahezu alle Schadstoffe. Ein generelles Ziel der Regenwasserbehandlung liegt damit im möglichst effizienten Rückhalt von Feststoffen. Dies aber erfordert hinreichend zuverlässige Informationen zu Menge und Qualität der in Entwässerungssystemen transportierten Feststoffe.

\subsection{Feststoffmenge}

Die Bandbreite der dokumentierten Feststoffkonzentrationen beträgt nicht selten mehrere Zehnerpotenzen, wie die Darstellung der in Mitteleuropa beobachteten Messwerte für AFS im Wahrscheinlichkeitsplot verdeutlicht (Abb. 1).

Auch ohne detaillierte Kenntnisse zu den Einzugsgebieten und Untersuchungsmethoden der einzelnen Programme kann davon ausgegangen werden, dass das breite Wertespektrum primär auf differierende Einzugsgebietseigenschaften zurückzuführen ist. Es kann weiterhin unterstellt werden, dass Besonderheiten der Entwässerungssysteme Einfluss auf das Konzentrationsniveau haben.

Selbst eine kleinräumigere oder detailliertere Betrachtung von mittleren Regenabflusskonzentrationen reduziert die Variabilität der Daten zunächst nicht. Tabelle 1 zeigt die Bandbreite spezifischer Feststoffausträge verschiedener Einzugsgebiete insbesondere im Stadtgebiet von Berlin.

Die Daten der ersten fünf Zeilen sind das Ergebnis von Untersuchungen an Straßen. An der A100 und A113 (neu) wurden jeweils vier aufeinanderfolgende Straßeneinläufe (Nassgully) beprobt. Die nachfolgenden Beispiele resultieren aus größeren, zusammenhängenden innerstädtischen Einzugsgebieten. 
Tab. 1 Bandbreite der spezifischen Feststofffrachten in unterschiedlichen Einzugsgebieten

\begin{tabular}{llcc}
\hline Quelle & EZG & $\mathbf{A}_{\mathbf{u}}$ in ha & AFS in $\mathbf{~ k g} /(\mathbf{h a}$ a) \\
\hline Eignen Messungen $^{\mathrm{a}}$ (2009) & Berlin, A 113 (neu) & 0,018 & 5927 \\
Eignen Messungen $^{\mathrm{a}}$ (2009) & Berlin, A100 & 0,011 & 4560 \\
Pick et al. (2002) & Höxter & 0,030 & 2512 \\
Grottker (1987) & Hildesheim & 0,410 & 2100 \\
Nadler und Meißner (2004) & Augsburg & 0,001 & 1300 \\
Fuchs (2008) & Berlin, Fennsee & 90 & 1130 \\
Grotehusmann et al. (2005) & Berlin, Adlershof & 45,0 & 344 \\
Terzioglu et al. (1987) & Berlin, Wilmersdorf & 12,7 & 337 \\
Lambert et al. (2005) & Berlin, Biesdorf & 50,8 & 327 \\
Schütte (2001) & Berlin, Rudower Felder & 14,1 & 305 \\
Heinzmann (1993) & Berlin, Reinickendorf & 12,6 & 212 \\
Grotehusmann et al. (2009) & Berlin, Pücklerteich & 23 & 103 \\
\hline
\end{tabular}

${ }^{a}$ Untersuchungen zum Feststoffrückhalt von Nassgullys im Auftrag der Berliner Wasserbetriebe.
In Verbindung mit den neben der Feststofffracht erhobenen Gebietskenndaten können aus den in Tab. 1 aufgelisteten Daten die folgenden grundsätzlichen Zusammenhänge abgeleitet werden:

- Sehr hohe spezifische Feststofffrachten treten in der Regel nur in kleinen Einzugsgebieten auf, in denen Retentions- und Klassierungsprozesse von untergeordneter Bedeutung sind (z. B. Straßeneinzugsgebiete).

- Treten sehr hohe Frachten auf, so ist am Probenahmeort das gesamte auf den Oberflächen vorhandene Korngrößenspektrum (feines und grobes Material) anzutreffen.

- Eine deutliche Reduzierung der Feststofffrachten tritt häufig in größeren urbanen Entwässerungsnetzen auf und weist auf Retentionsprozesse hin, die immer mit einer Kornklassierung verbunden sind. Am Probenahmeort wird vor allem feines Material im Niederschlagsabfluss zu finden sein.

- Werden in größeren innerstädtischen Einzugsgebieten hohe spezifische Feststofffrachten erreicht, so ist dies ein Hinweis auf einen bedeutenden Vegetationseinfluss.

Zusammenfassend kann festgehalten werden, dass ortsspezifisch immer hohe Konzentrationsschwankungen auftreten, die insbesondere auf saisonale Effekte (z.B. Baumblüte, Streusalzanwendung) und Wechselwirkungen im Einzugsgebiet sowie in der Kanalisation zurückzuführen sind.

Während über den saisonalen Einfluss vor allem das Stoffdargebot im Einzugsgebiet beeinflusst wird, bestimmen die Transport-, Retentions- und Umsatzprozesse auf den befestigten Oberflächen und im Kanal, welcher Anteil und welche Fraktion der Feststoffe an einer Einleitstelle oder am Behandlungsort anfallen.

\subsection{Feststoffeigenschaften}

Im Hinblick auf die effektive Behandlung von Siedlungsabflüssen ist eine weitere Differenzierung der Feststoffe in eine feine $(\mathrm{T}+\mathrm{U}){ }^{-1}$ und grobe $(\mathrm{S}+\mathrm{G})$-Fraktion erforderlich. In Abb. 2 sind zunächst die spezifischen Sand- und Kiesfrachten in 13 Berliner Einzugsgebieten dargestellt (Fuchs et al. 2009b).

Die einzugsgebietsspezifischen Grobpartikelfrachten in diesen Gebieten schwanken um mehr als den Faktor 5000 zwischen 0,8 und $5.465 \mathrm{~kg} /$ (ha a). Der Gesamtanfall von Grobpartikeln, wie auch die Bedeutung des durch die Stadtreinigung entnommenen Feststoffanteils, kann durch Gullyuntersuchungen an der A100 und A113 (neu) illustriert werden. Die Autobahneinzugsgebiete, die durch hohe Seitenbegrenzungen und Lärmschutzwände von der Umgebung abgeschlossen sind, repräsentieren mit einer spezifischen Jahresfracht von bis zu $6000 \mathrm{~kg} /$ (ha a) das mineralische (GV 6,4 bis 8,6\%) Grobkornpotenzial. Diese hohe Grobstofffracht stammt aus dem Fahrbahnabrieb und wird über die Gullyräumung nahezu quantitativ aus dem Einzugsgebiet entfernt. Die ebenfalls anfallenden Feinpartikel werden dagegen durch die Nassgullys hindurch zum Gewässer resp. zur Behandlungsanlage transportiert. Der Feinkornanteil in den Gullys liegt nur zwischen 5,1 und 7,8\%.

Verallgemeinernd kann festgehalten werden, dass an Orten geringer Grobpartikelkonzentrationen der überwiegende Anteil von Sand und Kies über die Straßen-, Gully- und Kanalreinigung und nicht durch den Regenabfluss aus dem Gebiet entfernt wird.

Im Gegensatz zur groben Fraktion schwankt die über den Regenabfluss aus den Gebieten heraus transportierte feinpartikuläre Fracht ausgesprochen wenig. Die Feinpartikelfracht in den bisher untersuchten Gebieten liegt zwischen 200 und $400 \mathrm{~kg} /$ (ha a) und ist damit im Vergleich zu den Grobstoffen gut kalkulierbar. Treten deutlich geringere

\footnotetext{
${ }^{1}$ Die Begriffe T+U (Ton und Schluff) wie auch $\mathrm{S}+\mathrm{G}$ (Sand und Kies) beziehen sich ausschließlich auf die Korngröße der Partikel und nicht auf deren chemisch-physikalische Eigenschaften.
} 
Abb. 2 Bandbreite der spezifischen Feststofffrachten $(\mathrm{S}+\mathrm{G})$ in unterschiedlichen Berliner Einzugsgebieten $(*=$ Probenahme im Gully)

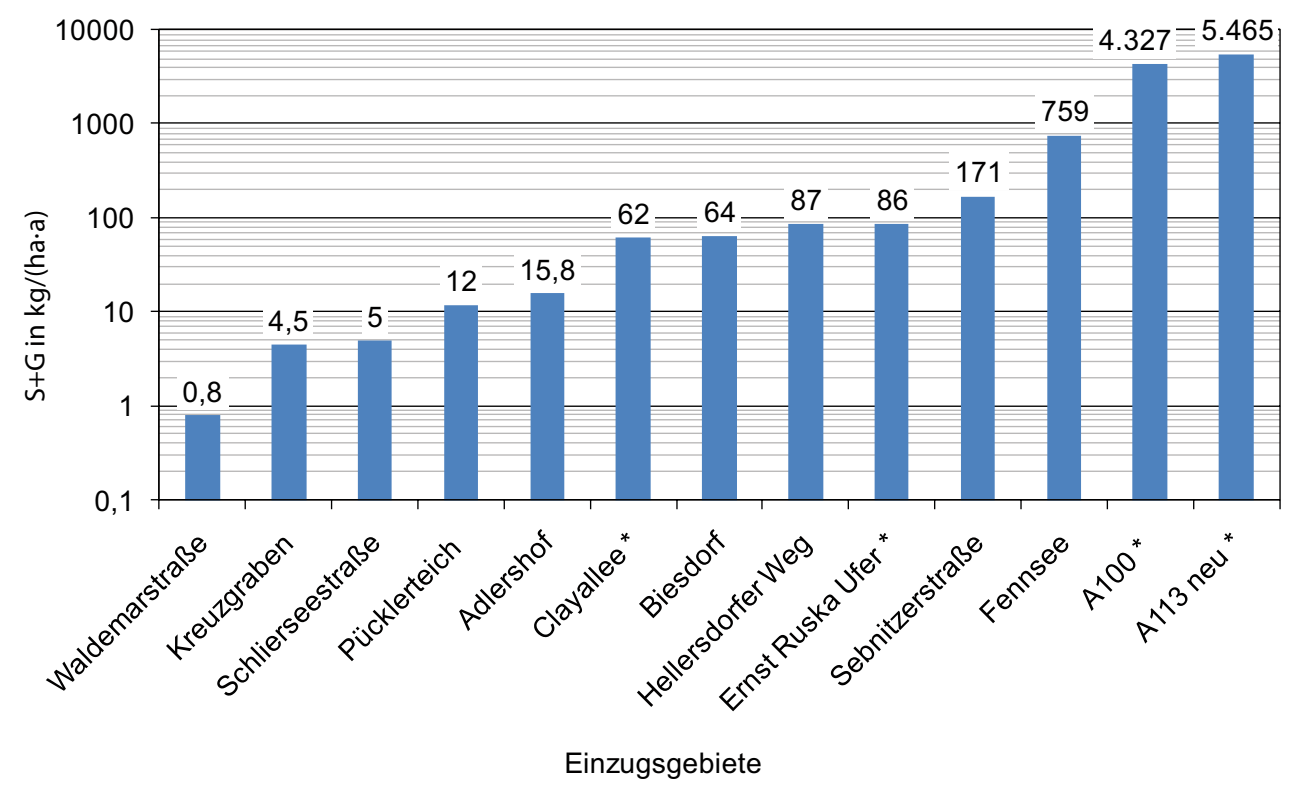

Tab. 2 Spezifische Grob- und Feinpartikelfracht in Berliner Einzugsgebieten

\begin{tabular}{lcc}
\hline Einzugsgebiet & S+G in $\mathbf{~ k g} /(\mathbf{h a}$ a) & $\mathbf{T}+\mathbf{U}$ in $\mathbf{~ k g} /(\mathbf{h a}$ a) \\
\hline Adlershof & 16 & 264 \\
Biesdorf & 64 & 203 \\
Fennsee & 759 & 370 \\
Pücklerteich & 12 & 91 \\
\hline
\end{tabular}

Feinpartikelfrachten am Gebietsauslass auf, wie am Beispiel Pücklerteich (Tab. 2), so ist dies auf besondere, ortsspezifische Bedingungen (Pflasterstraßen, häufige Kehrzyklen) zurückzuführen.

Diese Feinpartikelfracht, die unter den mittleren Berliner Abflussbedingungen einer Konzentration von 50 bis $100 \mathrm{mg} / 1$ entspricht, fällt in urbanen Einzugsgebieten immer an und wird durch die grobe Fraktion mehr oder minder aufgestockt.

Eine Ausnahme hiervon bilden Einzugsgebiete mit einer Muldenentwässerung oder mit einem hohen Retentionspotenzial auf den befestigten Flächen. Eine weitere Besonderheit mit Einfluss auf die Feinpartikelkonzentration im Regenabfluss sind eingestaute Kanäle. Sie wirken bei einer Vielzahl von Regenereignissen wie großvolumige Sedimentationsanlagen und sind daher temporäre Stoffsenken. Tabelle 2 zeigt neben den geringen Schwankungen der spezifischen Feinpartikelfracht, dass in den untersuchten Einzugsgebieten der überwiegende Anteil der am Gebietsauslass ankommenden Feststoffe der feinen Fraktion zuzurechnen ist. Eine Ausnahme hiervon ist das Einzugsgebiet Fennsee, in dem aufgrund eines sehr hohen Baumbestandes Vegetationsabfälle (Holz) die Grobfraktion erheblich aufstocken.
Tab. 3 Eigenschaften der Feststoffe im Regenabfluss

\begin{tabular}{lccc}
\hline & T+U & S+G mineralisch & S+G organisch \\
\hline GV in $\%$ & 40,0 & 2,50 & 90,0 \\
$\mathrm{P}$ in $\mathrm{mg} / \mathrm{kg}$ & 2,90 & 0,20 & 0,86 \\
$\mathrm{Zn}$ in $\mathrm{mg} / \mathrm{kg}$ & 2.915 & 150 & 1.169 \\
$\mathrm{Fe}$ in $\mathrm{mg} / \mathrm{kg}$ & 25,9 & 5,00 & 8,81 \\
\hline
\end{tabular}

Neben der Feststoffmenge und Korngröße wurden die Feststoffe im Regenabfluss auch bezüglich ihrer Stoffbeladung charakterisiert. In Tab. 3 sind die hierbei gewonnenen Ergebnisse beispielhaft zusammengefasst.

Es zeigt sich, dass der Summenparameter AFS über die Eigenschaften Korngröße und Glühverlust weiter zu differenzieren ist, um einen Bezug zu Gewässerschutz- und Reinigungszielen herstellen zu können. Es ergeben sich drei unterschiedlich zu bewertende Fraktionen:

1. Die Feinfraktion $(\mathrm{T}+\mathrm{U})$, die immer hochbelastet und schwer absetzbar ist.

2. Die mineralische Grobfraktion $(\mathrm{S}+\mathrm{G})$, die stets gering oder unbelastet und sehr leicht absetzbar ist.

3. Die organische Grobfraktion, die eine nennenswerte Belastung trägt und primär schwer absetzbar ist. Sie kann als Schwimm- oder Schwebstoff im Regenabfluss auftreten. Zusätzlich ist zu beachten, dass sich diese Grobfraktion im Entwässerungssystem nicht konservativ verhält, sondern im Laufe der Zeit weitgehend aufgearbeitet wird, sodass ein Wandel von grob nach fein stattfindet.

Zur wirksamen stofflichen Entlastung der Gewässer sind grundsätzlich die Feinpartikel, und, wo vorhanden, auch der organische Teil der groben Fraktion mit hohem Wirkungsgrad zurückzuhalten. 
Aus der Zusammenfassung der Befunde zu Menge und Qualität von Feststoffen in Entwässerungssystemen ergeben sich weitere Schlussfolgerungen:

1. Hohe AFS-Konzentrationen im Regenabfluss weisen im statistischen Mittel auf eine breite Korngrößenverteilung bzw. nennenswerte Grobkornanteile hin.

2. Geringe AFS-Konzentrationen sind immer mit hohen Fein- und zunehmenden Organikanteilen verbunden.

3. Die Anreicherung der Feinpartikel im Niederschlagsabfluss ist mit einer beträchtlichen Erhöhung der spezifischen Schadstoffkonzentration verbunden.

\section{Wirksamkeit verschiedener Instrumente der Regenwasserbehandlung}

Die verfügbaren und flächendeckend umgesetzten Instrumente der Regenabflussbehandlung beziehen sich in der Regel nicht auf weitergehende Reinigungsziele. Bisher wurden deshalb vor allem Absetzverfahren realisiert, die allerdings für die gewässerschutzrelevanten Feinpartikel $(<63 \mu \mathrm{m})$ keinen oder nur einen geringen Wirkungsgrad aufweisen. In den vergangenen 15 Jahren wurden in Deutschland vermehrt Filtrationsverfahren in Form der Retentionsbodenfilter eingesetzt. Diese halten im Gegensatz zu den Absetzverfahren die Feinpartikel weitgehend (>90\%) zurück.

\subsection{Konventionelle Absetzanlagen}

In Abb. 3 ist die Wirksamkeit verschiedener Absetz- und Rückhalteanlagen anhand der erreichten mittleren AFSAblaufkonzentrationen dargestellt. Es wird deutlich, dass insbesondere die Anlagen mit einem Volumen von weniger als $50 \mathrm{~m}^{3} /$ ha teilweise sehr hohe Ablaufkonzentrationen aufweisen. Die in der Regel realisierte Beckengröße beträgt ca. $10 \mathrm{~m}^{3} / \mathrm{ha}$. Aber auch großvolumige Regenklärbecken $\left(50 \mathrm{~m}^{3} / \mathrm{ha}\right)$ sind in Bezug auf die Feinpartikel leistungsschwach. Unterstellt man beispielsweise eine spezifische Feinpartikeljahresfracht von $300 \mathrm{~kg} /$ (ha a) und einen mittleren Jahresabfluss von $3870 \mathrm{~m}^{3}$ (ha a) (Rechenwert für Berlin nach Rehfeld-Klein 2001), so wird klar, dass ein Feinpartikelrückhalt in Anlagen mit einem spezifischen Volumen von $<50 \mathrm{~m}^{3} /$ ha quasi nicht stattfindet.

Nur bei Anlagen mit einem deutlich höheren spezifischen Volumen $\left(>100 \mathrm{~m}^{3} / \mathrm{ha}\right)$ kann von einem sicheren Feinpartikelrückhalt ausgegangen werden. Hierbei handelt es sich allerdings um Regenrückhalteanlagen, deren Zweck nicht der Stoffrückhalt, sondern ausschließlich die Abflussretention ist.

\subsection{Retentionsbodenfilter}

Retentionsbodenfilter werden in zunehmendem Maße eingesetzt, wenn hohe oder weitergehende Anforderungen an die Regenwasserbehandlung gestellt werden. Ihre Notwendigkeit leitet sich damit von gewässerseitigen Anforderungen ab.

Der Feststoffrückhalt von Retentionsbodenfiltern ist nahezu quantitativ, sodass Filtrationsanlagen auch eine hohe Wirksamkeit für anthropogene Schadstoffe, wie Schwermetalle, PAK und MKW, aufweisen.

Grundsätzlich gilt für die anorganischen und organischen Schadstoffkomponenten, dass sie, abgesehen von Unfallereignissen, dominant mit den feinen Feststoffen transportiert werden. Tabelle 4 zeigt die mit Retentionsbodenfiltern erreichbaren Ablaufkonzentrationen.

Aufgrund ihres hohen Flächenbedarfs sind Retentionsbodenfilter insbesondere im urbanen Raum jedoch nur be-
Abb. 3 AFS-Ablaufkonzentrationen verschiedener Absetzund Rückhalteanlagen

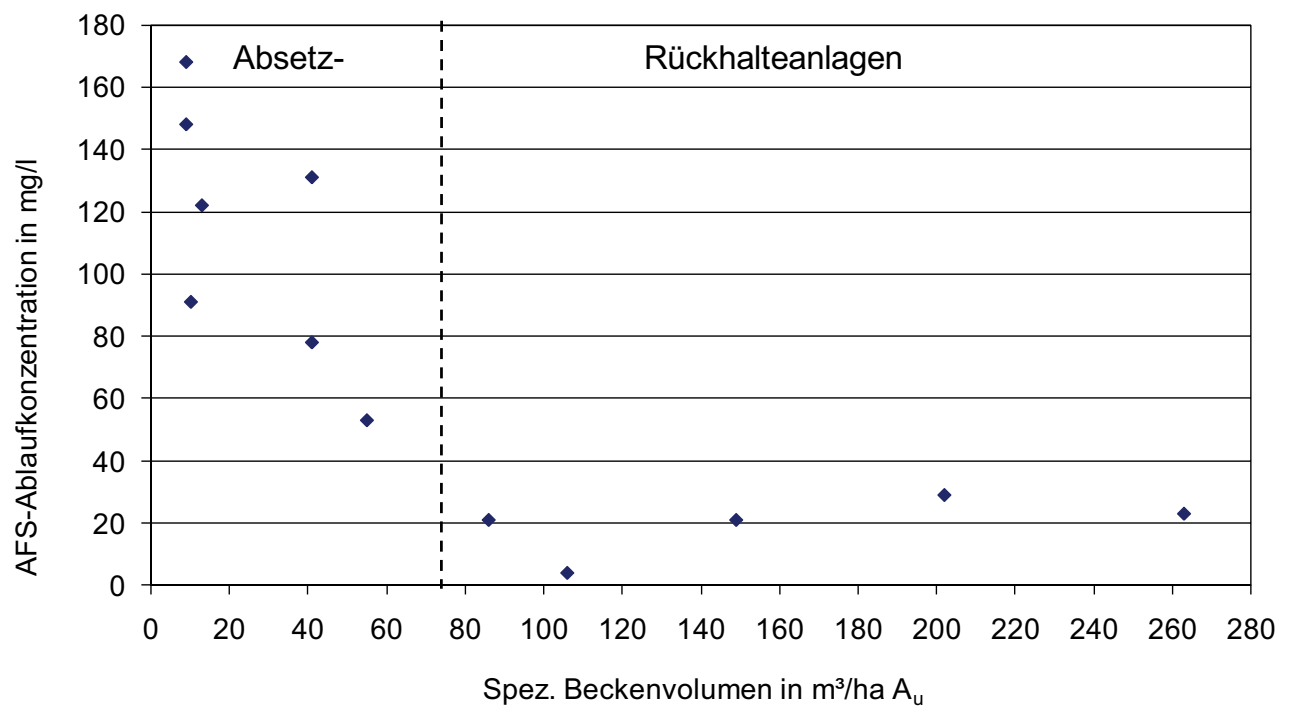


Tab. $4 \mathrm{Zu}$ - und Ablaufkonzentrationen von Bodenfiltern mit melioriertem Filtersubstrat

\begin{tabular}{lcc}
\hline & Zulauf in $\mathbf{~ m g / l}$ & Ablauf in $\mathbf{~ m g / l}$ \\
\hline $\mathrm{AFS}$ & 149 & $<1$ \\
$\mathrm{CSB}_{\text {ges }}$ & 82,9 & 16,7 \\
$\mathrm{BSB}_{5}$ & 12,5 & 1,4 \\
$\mathrm{P}_{\text {ges }}$ & 0,44 & 0,03 \\
$\mathrm{P}_{\text {gel }}$ & 0,06 & 0,03 \\
\hline
\end{tabular}

grenzt einsetzbar. Planung, Bau und Betrieb der Anlagen sind im Vergleich zu Absetzanlagen äußerst anspruchsvoll.

Der effektive Einsatz von Retentionsbodenfiltern erfordert darüber hinaus, mehr als konventionelle Absetzanlagen, genaue Kenntnisse zu den Eigenschaften der Einzugsgebiete. Vordergründig bezieht sich dies auf die zu behandelnde Wassermenge. Eine Überschätzung der Zuflussmenge führt $\mathrm{zu}$ erheblichen Betriebsproblemen (z.B. Etablierung des Schilfbewuchses) und Minderleistungen (fehlende Konditionierung der Mikroorganismen). Letztlich kann eine dauerhaft unterbelastete Anlage durch Tierbauten, den Ausfall der Schilfvegetation und das Aufkommen von Gehölzen zerstört werden.

Eine Unterschätzung der Zuflussmenge führt zur hydraulischen und stofflichen Überlastung und, wie in zahlreichen Fällen dokumentiert, ebenfalls zum Totalausfall der Anlage durch Kolmation.

\subsection{Anlagen mit verbesserter Sedimentationsleistung}

Aus der geringen Wirksamkeit von konventionellen Absetzanlagen und den Restriktionen für den Einsatz von Retentionsbodenfiltern ergibt sich insbesondere im innerstädtischen Raum der Bedarf zur Entwicklung neuer und effizienter Methoden zur Regenabflussbehandlung.

Die Nachrüstung von Schrägklärern kann bei gleichem Volumen der Absetzanlage eine erhebliche Verbesserung der Sedimentationsleistung in Bezug auf die Zielgröße Feinpartikel bewirken. Abbildung 4 zeigt zwei Einbausitua- tionen von Platten- resp. Röhrenabscheidern. Die gezeigten Anlagen wurden über einen Zeitraum von zwei Jahren untersucht (Fuchs et al. 2010).

Bei regelgerechtem Betrieb der Anlagen wurden bei mittleren AFS-Wirkungsgraden zwischen 58 und $68 \%$ AFS-Ablaufkonzentrationen von 60 bis $70 \mathrm{mg} / 1$ gemessen. Bemerkenswert ist, dass bei allen Anlagen auch für die partikuläre Schadstoffphase ein Wirkungsgrad in gleicher Größenordnung erreicht wurde. Unter Berücksichtigung der in Abschn. 2 dargelegten Zusammenhänge bedeutet dies, dass die gemessenen Feststoffrückhalte tatsächlich auf Feinpartikel zu beziehen sind.

Dies kann erreicht werden, da durch den Einbau der Schrägklärelemente die tatsächlich zur Verfügung stehende Sedimentationsfläche um den Faktor 5 bis 10 erhöht oder umgekehrt die Oberflächenbeschickung in gleicher Höhe reduziert wird. Voraussetzung für eine hohe Leistung von Schrägklärelementen ist, dass Grobstoffe vor der Anlage abgeschieden werden und dass eine gleichmäßige Durchströmung des Schrägklärers sichergestellt ist.

Eine weitere Möglichkeit der effizienten Regenwasserbehandlung ergibt sich aus der Nutzung der in urbanen Räumen häufig anzutreffenden Regenrückhalteanlagen. Wie in Abb. 3 deutlich wird, haben diese Anlagen aufgrund ihres hohen spezifischen Volumens eine gute Sedimentationsleistung, die nicht systematisch genutzt wird.

Durch geringe bauliche Maßnahmen können diese Regenrückhalteanlagen $\mathrm{zu}$ Hochleistungssedimentationsanlagen umgebaut werden, wie Abb. 5 beispielhaft illustriert (Lemm et al. 2009).

Die zentrale Maßnahme ist im Einbau einer Ablaufsteuerung zu sehen, die einen gezielten Betrieb der Anlage erlaubt. Bei Beschickung der Anlage ist der Ablauf zunächst geschlossen, sodass immer ein Einstau stattfindet. Nach einer vorwählbaren Absetzzeit (mehrere Stunden) wird der Ablauf geöffnet und das weitgehend partikelfreie Einstauwasser wird abgeleitet. Aufgrund der hohen Volumen der Becken und der gegebenen Niederschlagsverteilung kann auf diese Weise ein hydraulischer Wirkungsgrad von $>90$ erreicht werden. Das bei wenigen Großereignissen über-
Abb. 4 Schrägklärelemente in einem Regenklärbecken (links) und einem Regenüberlaufbecken (rechts)
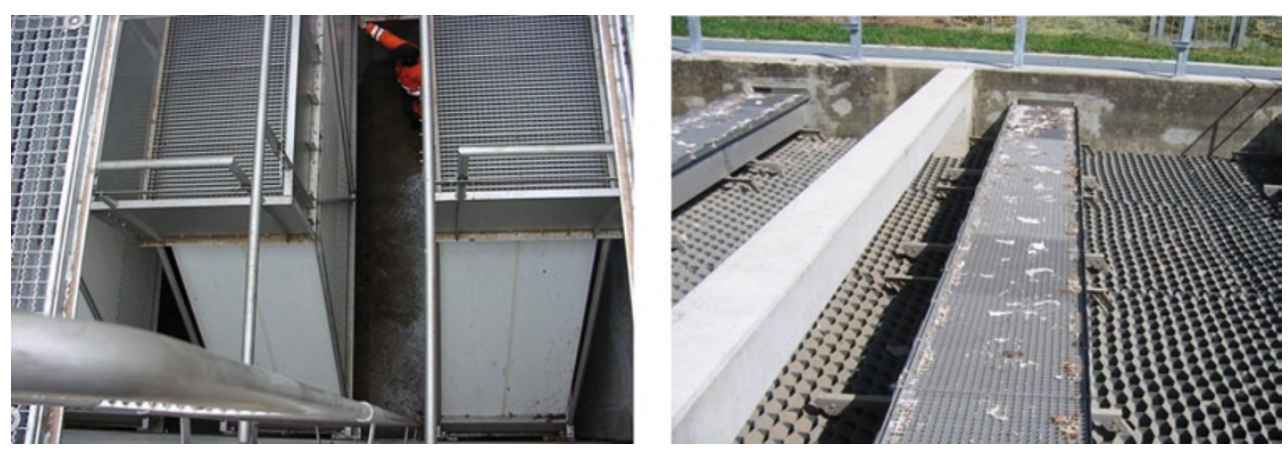
Abb. 5 Schemaskizze einer Hochleistungssedimentationsanlage

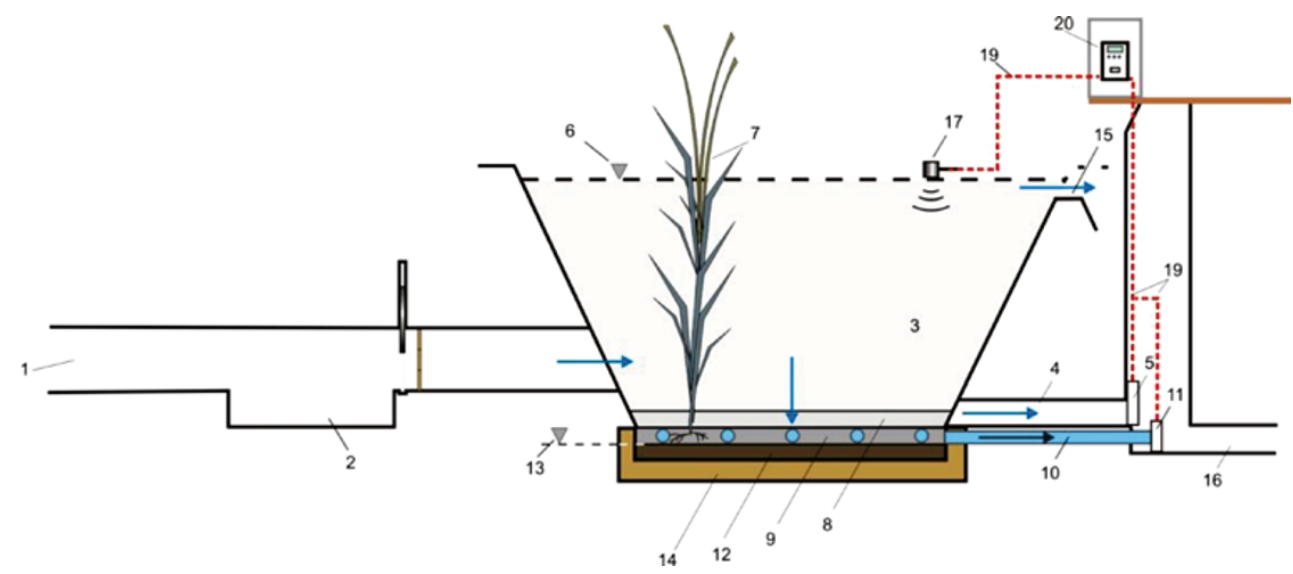

laufende Wasser wird durch die Durchströmung des schilfbewachsenen Retentionsraumes ebenfalls gereinigt. Der grundsätzlich hohe Partikel- und Stoffrückhalt dieses neuartigen Anlagentyps wird derzeit in einer Pilotanlage in Berlin quantifiziert.

\section{Literatur}

Brombach H, Fuchs S (2002) Datenpool gemessener Verschmutzungskonzentrationen von Trocken- und Regenwetterabflüssen in Misch- und Trennkanalisationen. Abschlussbericht, ATVDVWK-Forschungsfond-Vorhaben 1/01, ATV-DVWK, Hennef

Fuchs S (2008) Maßnahmenplanung Regenwasser. In: Stoffströme in Flussgebieten - von der Bilanzierung zur Bewirtschaftung. Institut für Wasser und Gewässerentwicklung, Bereich Siedlungswasserwirtschaft und Wassergütewirtschaft, Bd 128

Fuchs S, Scherer U, Wander R, Behrendt H, Venohr M, Opitz D, Hillenbrandt T, Marscheider-Weidemann F, Götz T (2009a) Modellbasierte Quantifizierung und Internet-basierte Visualisierung der Stoffeinträge in die Fließgewässer Deutschlands. Nährstoffe, Schwermetalle und weitere prioritäre Stoffe. Abschlussbericht zum UBA-Vorhaben FKZ 20424218

Fuchs S, Lambert B, Grotehusmann D, Lemm K (2009b) Einflussmöglichkeiten der Regenwasserbehandlung auf die Gewässergüte am Beispiel des Berliner Gewässersystems. DWA 8. Regenwassertage, 27./28.5.2009, Dortmund

Fuchs S, Mayer I, Haller B, Roth, H (2010) Einsatz von Schrägklärern in der Regenwasserbehandlung. Korresp Abwasser, zur Veröffentlichung angenommen
Grotehusmann D, Lambert B, Fuchs S (2005) Betriebsbegleitende Untersuchung des Retentionsbodenfilters Adlershof. Zwischenbericht, Auftraggeber Berliner Wasserbetriebe, Abtlg NB-G

Grotehusmann D, Lambert B, Fuchs S (2009) Regenwasserbehandlung Pücklerteich. Messprogramm zur Erfassung der Ausgangsbelastung und Konzeption der Regenwasserbehandlung. Abschlussbericht, Auftraggeber Berliner Wasserbetriebe, Abtlg NB-G

Grottker M (1987) Regenwasserbehandlung im Trennsystem. Dissertation, Universität Hannover

Heinzmann B (1993) Beschaffenheit und weitergehende Aufbereitung von städtischen Regenabflüssen. Fortschrittsberichte VDI, Reihe $15, \mathrm{Nr} 113$, VDI

Lambert B, Grotehusmann D, Fuchs S (2005) Retentionsbodenfilter Biesdorf. Zwischenbericht der 2. Untersuchungsphase. Auftraggeber Berliner Wasserbetriebe, unveröffentlicht

Lemm K, Joswig K, Fuchs S, Lambert B, Grotehusmann D (2009) Verfahren zur Behandlung von Regenwasser und Vorrichtung zur Durchführung des Verfahrens. Patentschrift 102005021816

Nadler A, Meißner E (2004) Versickerung des Niederschlagswassers von befestigten Verkehrsflächen. 3. Zwischenbericht. Bayerisches Landesamt für Wasserwirtschaft, München

Pick V, Fettig J, Miethe M, Stüben K (2002) Dezentrale Behandlung des Niederschlagsabflusses von Verkehrsflächen in einer Schachtanlage. Korresp Abwasser 49:312-320

Rehfeld-Klein M (2001) Abwasserbeseitigungsplan Berlin, 1. Aufl. Senatsverwaltung für Stadtentwicklung, Berlin

Schütte M (2001) Güte- und Mengenmessungen am Regenüberlaufbecken Rudower Felder. Auftraggeber Berliner Wasserbetriebe, IfS Hannover

Terzioglu K, Peter A, Sarfert F (1987) Regenklärbecken am Dianasee in Berlin. Korresp Abwasser 34:1216-1302 